\title{
Manger la parole de Dieu. La Bible lue par les Wari'
}

\section{Aparecida Vilaça}

\section{OpenEdition}

\section{Journals}

Édition électronique

URL : https://journals.openedition.org/jsa/12154

DOI : 10.4000/jsa. 12154

ISSN : 1957-7842

\section{Éditeur}

Société des américanistes

\section{Édition imprimée}

Date de publication : 31 juillet 2012

Pagination : 81-100

ISSN : 0037-9174

\section{Référence électronique}

Aparecida Vilaça, "Manger la parole de Dieu. La Bible lue par les Wari' », Journal de la Société des américanistes [En ligne], 98-1 | 2012, mis en ligne le 10 juin 2015, consulté le 03 septembre 2022. URL http://journals.openedition.org/jsa/12154 ; DOl : https://doi.org/10.4000/jsa.12154 


\title{
MANGER LA PAROLE DE DIEU. LA BIBLE LUE PAR LES WARI'
}

\author{
Aparecida VILAÇA *
}

L'objectif de cet article est d'analyser un aspect particulier de l'expérience chrétienne chez les Wari' (Amazonie, Brésil) : leur intérêt pour la Bible, traduite dans leur langue par les missionnaires de la Mission Nouvelles Tribus qui vivent depuis près de cinquante ans parmi eux. La conception de la Parole de Dieu comme aliment permet en quelque sorte d'assimiler la lecture et l'écoute de la Bible aux autres modalités indigènes de production des relations de parenté. Ce processus est encore renforcé par le contenu moral des paroles divines, ainsi que par leurs prémisses ontologiques qui permettent de différencier clairement l'humain de l'animal, confortant ainsi la position humaine des Wari'. [Mots-clés : Amazonie, christianisme, la Bible, Wari'.]

Comendo a palavra de Deus : a Bíblia na leitura dos Wari'. O artigo tem por objetivo analisar um aspecto particular da experiência cristã dos Wari' (Amazônia, Brasil) : o seu interesse pela Bíblia, traduzida no idioma nativo por missionários protestantes da Missão Novas Tribos do Brasil com quem convivem há cerca de cinquenta anos. A concepção da palavra de Deus como alimento possibilita equacionarem a relação de leitura e escuta ao modo nativo de produção de parentesco, processo este que se vê reforçado pelo conteúdo moral das palavras divinas, assim como por suas premissas ontológicas, que ao diferenciarem claramente a humanidade da animalidade, permitem que os Wari' estabilizem-se na posição de humanos. [Palavras chaves : Amazônia, cristianismo, Bíblia, Wari'.]

Eating God's Words : the Bible as read by the Wari'. This paper deals with one particular aspect of Christianity as experienced by the Wari' (Amazonia, Brazil) : the interest they take in the Bible, which has been translated into their language by the New Tribes missionaries who have been living among them for nearly fifty years now. The comparison of God's Words with food equates reading or hearing them with other native ways of producing kinship. This process is reinforced by the moral contents of the Holy Words, as well as by their ontological premises, since the clear-cut distinction between human and non-human animals helps to ratify the Wari's human status. [Key words : Amazonia, christianity, Bible, Wari'.]

* PPGAS-Museu Nacional-UFRJ, Quinta da Boa Vista s/nº, São Cristóvão, 20940-040 Rio de Janeiro, Brasil [aparecida.vilaca@terra.com.br].

Journal de la Société des Américanistes, 2012, 98-1, pp. 81-100. (C) Société des Américanistes. 
La réflexion qui est développée ci-dessous m'a été inspirée par un événement précis. En février 2008, Abrão, mon frère wari', est venu avec moi à Rio pour suivre un traitement médical. Il est resté chez moi pendant un mois et demi. Abrão, qui, enfant, avait été « croyant », s'est converti au christianisme protestant une nouvelle fois, l'année dernière, dans des circonstances particulières : gravement invalidé par une infection qui avait provoqué de l'arthrose, il a été soutenu à l'hôpital par un groupe de pasteurs protestants qui ont prié pour sa guérison pendant plusieurs jours. Depuis lors, Abrão, âgé de 40 ans et professeur indigène, est devenu un croyant consciencieux, qui assiste aux cultes avec sa Bible (en portugais) en mains et fait répéter un petit chœur familial. Il m'a dit lire souvent la Bible à sa famille.

Dans ses bagages pour venir à Rio, il avait emporté, en plus de ses vêtements et d'un magnétophone, sa Bible et son livre d'hymnes. Son séjour n'a pas été facile car, son état de santé l'empêchant de se déplacer, il restait seul chez moi pendant que j'allais travailler. La télévision, me disait-il, le fatiguait au bout d'un moment. Pour l'aider, je lui proposais les nombreux livres de leçons chrétiennes publiés par la New Tribes Mission en langue wari', qu'il connaissait déjà. Pourtant il n'ouvrit aucun de ces livres, ni même la Bible. Cela m'interpella car, d'après les protestants occidentaux que j'ai pu rencontrer, la lecture de la Bible leur apporte un réconfort émotionnel en permettant une relation directe avec Dieu. Je me demandais pourquoi Abrão, qui connaît aussi bien le wari' que le portugais, ne souhaitait pas lire la Bible pour trouver, de cette façon, le réconfort de se sentir proche de Dieu, alors même qu'il traversait une période de grande souffrance physique loin de ses parents, seul dans une maison inconnue. Tout changea lorsque je lui demandai de travailler à la transcription de deux cultes que j'avais enregistrés dans l'église du village de Rio Negro-Ocaia. Nous avions recours aux livres de leçons en langue wari' et à la Bible en portugais pour vérifier les traductions et discuter du contenu des passages de la Bible. Dès lors, Abrão commença à lire sa Bible, même en mon absence. Il lisait à voix haute, en particulier dans mon bureau où l'on travaillait ensemble et où il dormait.

Le but principal de ces lignes est de tenter de comprendre l'intérêt manifesté par les Wari' pour la Bible et les livres religieux, à l'exclusion d'autres écrits, et d'analyser la façon dont ils conçoivent la capacité médiatrice de la parole de Dieu.

\section{UNE BRÈVE HISTOIRE DE LA RENCONTRE AVEC LES MISSIONNAIRES}

Les Wari', plus anciennement connus dans la littérature comme Pakaa Nova ou Pacaás Novos, parlent une langue rattachée à la famille linguistique chapakura et habitent à l'ouest de l'État du Rondônia, en Amazonie brésilienne. Ils sont, aujourd'hui, près de 3000 , répartis dans 23 villages, situés en majorité le long de la rivière Pacaás Novos et de ses affluents, lieu de résidence wari’ très ancien. Wari' 
est le nom qu'ils utilisent pour se présenter aujourd'hui aux Blancs. Il signifie « nous ", « être humain », " gens ». Ils doivent leur renommée nationale à la médiatisation de leurs activités guerrières, ainsi qu'à leur pratique du cannibalisme (funéraire et guerrier). Avant 1956, ils n'avaient jamais eu de contacts pacifiques avec les Blancs qu'ils désignaient alors, comme aujourd'hui, par le terme wijam, « ennemi ».

Dans les années 1940, le gouvernement brésilien, par l'intermédiaire du Service de protection des Indiens (SPI), a commencé à organiser des expéditions de contact ayant pour objectif de les « pacifier » et de les amener à participer à la vie nationale (Vilaça 1992, 2006, 2010). Ces expéditions n'eurent de succès que grâce à la participation des missionnaires fondamentalistes de la New Tribes Mission (NTM), au début des années 1950. En 1956, le premier contact pacifique eut lieu avec les Wari' du sous-groupe OroNao' ${ }^{1}$, qui vivaient sur les affluents de la rive gauche du Pacaás Novos. En 1961, la quasi-totalité des Wari' acceptait la proximité des Blancs. Bien que ces missionnaires eussent appuyé leur action par des dons de biens matériels, ils apparaissaient surtout comme des aventuriers décidés et téméraires, qui se lançaient à la recherche des Indiens avec une obstination presque suicidaire, faisant totalement confiance, selon leurs propres dires, à la protection divine (pour une description plus détaillée, voir Vilaça 1996, 1997 ; pour le récit d'une expédition de contact de ce type chez les Yanomami, voir Dawson 2000). Après avoir établi le contact, les missionnaires se sont intéressés à la langue wari' et, dès les premiers temps de leur apprentissage, ils ont prêché la parole de Dieu. La lettre d'un missionnaire à un fonctionnaire gradé du SPI, datée de 1962, l'informait que « les abécédaires de la langue sont en cours de relecture » (documents microfilmés, archives du Museu do Indio, Rio de Janeiro).

Selon le missionnaire Royal Taylor (communication personnelle, 1992), les premières conversions n'ont commencé qu'en 1968 et n'ont touché que des membres du premier sous-groupe à avoir été contacté, à savoir les OroNao'. Les Wari' expliquent qu'ils se sont convertis en masse à partir de 1970 et sont restés chrétiens une décennie entière, pour quitter cette foi au début des années $1980^{2}$. Lors de mon premier séjour dans le village Rio Negro-Ocaia, en 1986, peu nombreux étaient ceux qui se disaient croyants. Les réunions religieuses se tenaient dans la maison du couple de missionnaires brésiliens, à l'intérieur du village, car il n'y avait pas d'église.

À la veille de l'an 2000 et avec les annonces de fin du monde prévue au tournant du millénaire, un nouveau mouvement de conversion a débuté. Il s'est consolidé en septembre 2001 à la suite de l'attaque du World Trade Center dont les Wari' ont pu voir les images à la télévision communautaire. Ces derniers ont eu peur que la fin du monde les prenne au dépourvu : non croyants, leur destin serait, en effet, celui du feu de l'enfer ${ }^{3}$. Depuis lors, une bonne partie de la population se déclare chrétienne et assiste aux cultes qui ont lieu quatre fois par semaine 
- en plus du jour de prière - dans les églises construites dans presque tous les villages. La plupart du temps, il n'y a pas de missionnaires et les cultes sont exclusivement célébrés par des pasteurs indigènes, en langue wari'.

Notons que les Wari' ne se parlent entre eux que dans leur propre langue, ils utilisent quelques termes en portugais pour désigner les objets ou les actions qui n'existaient pas avant (comme le papier, le moteur, l'argent, les chiffres...). Les personnes âgées, de plus de 70 ans, ne parlent, ni ne comprennent le portugais.

\section{LE MATÉRIEL CHRÉTIEN ÉCRIT}

L'interprétation littérale de la Bible est la caractéristique des fondamentalistes au sein du protestantisme (Crapazano 2000 ; Fernandes 1980 ; voir Harding 2001 pour la création du fondamentalisme à partir du débat états-unien autour de la Genèse). Le premier principe des « bases de la foi » de la New Tribes Mission y fait d'ailleurs référence : «On croit 1. Dans l'inspiration verbale et la divine autorité des Saintes Écritures » (http://novastribosdobrasil.org.br, consulté le 02/02/2012). D'après un missionnaire de la NTM, auteur du manuel didactique de catéchèse adopté officiellement par la mission, « La Bible [...] est la narration de l'histoire ancienne, selon la perspective de Dieu. C'est Dieu racontant les événements historiques depuis le début de l'histoire » (McIlwain 2003, p. 104). Cette narration a pu se réaliser par l'intermédiaire des prophètes, à qui Dieu avait « donné les messages qu'il désirait voir rédigés. Parfois, Dieu communique oralement; d'autres fois sous forme d'apparitions, ou encore, en introduisant simplement les messages dans leurs esprits. Dieu a fait en sorte que les prophètes écrivent exactement ce qu'Il leur disait » (ibid., p. 107). Cette histoire et les principes moraux qu'elle contient peuvent et doivent, grâce au recours à la traduction, être transmis à tout peuple et à toute personne. Ce qui explique la place centrale accordée à la traduction dans le travail des missions. C'est la raison pour laquelle les missionnaires commencent toujours, dans la mesure du possible, par travailler avec des informateurs indiens et font avec eux leurs premiers essais de traduction des textes bibliques.

Tout comme l'écriture biblique a été inspirée de façon divine (Segal 2003, pp. 217-218), le travail de traduction en langue indienne l'est aussi (Johnston 1985, pp. 221-224). Avant tout, le missionnaire doit prier, demander à Dieu qu'Il l'inspire dans son travail et qu'Il illumine les Indiens de sorte que ceux-ci reçoivent son message. Pour les Wari', bien qu'il n'y ait eu aucune entité divine ou autre instance supérieure avant l'arrivée des missionnaires, la compréhension de ce qui est dit n'est pas dans le contenu de la parole, mais dépend de la capacité d'écoute de l'auditeur. L'élément important de la socialisation des enfants consiste justement à développer cette capacité, tant pour les Wari' que pour d'autres groupes amazoniens (Seeger 1980, 1981 pour les Suyá). 
Dans les années 1990, un changement s'est produit dans la méthode catéchistique de la NTM, jusque-là fondée sur la présentation de thèmes spécifiques, comme l'amour, le pardon, le sacrifice, illustrés par des exemples tirés de différents livres bibliques. En effet, grâce aux observations et au travail de McIlwain, missionnaire aux Philippines - qui a été confronté à la difficulté de susciter de "véritables conversions" des autochtones car, selon lui, ils ne comprenaient pas le message biblique -, il est apparu nécessaire de faire connaître aux futurs croyants « l'œuvre historique » de Dieu, telle qu'elle est racontée dans la Bible. Son livre de méthodologie propose la préparation de leçons qui respectent l'ordre chronologique des livres de la Bible. Les 46 leçons de l'Ancien Testament doivent être présentées en deux livres, le troisième présentant le Nouveau Testament. Chaque leçon mêle des citations bibliques sur le thème en question (le premier concerne la transcendance divine et la Sainte-Trinité ; le deuxième porte sur la création du monde...) et des explications détaillées, répétitives et doctrinaires des passages bibliques. Chaque leçon se termine sur une dizaine de questions, suivies des « bonnes » réponses (McIlwain 2003).

Chez les Wari', comme chez d'autres groupes amazoniens approchés par la NTM (comme les Marubo), ces leçons servent d'outils principaux pour la réalisation des cultes. Elles sont publiées dans la langue indienne et respectent à la lettre les instructions du manuel de McIlwain, depuis l'ordre des thèmes jusqu'aux questions finales. Six livres de leçons sont pour le moment disponibles. Ils sont produits artisanalement : préparés sur un ordinateur, puis édités à partir d'une simple imprimante, enfin reliés à la main.

Dans le village de Rio Negro-Ocaia, chacun des cinq pasteurs est responsable d'un livre. Chacun se consacre à l'étude des parties de son livre (qui compte environ 200 pages) pendant la semaine, en le lisant chez lui à ses enfants et à son épouse. Les trois cultes organisés pendant un week-end donné (samedi soir, dimanche matin et dimanche soir) sont consacrés à un livre en particulier et dirigés par le pasteur qui en est le responsable. La semaine suivante, ce sera le tour d'un autre livre, donc d'un autre pasteur, selon un ordre qui va du livre 1 au livre 5, pour ensuite reprendre le livre 1 et ainsi de suite. Chaque pasteur choisit de façon aléatoire le passage de son livre à étudier durant le week-end. Il n'en continuera l'étude que quatre semaines plus tard, lorsque son tour reviendra.

Il existe également des livres qui rassemblent le résumé des thèmes abordés lors des cours de quelques jours offerts par les missionnaires de la NTM dans différents villages (notamment à l'époque de Noël). Ces "journées d'étude» réunissent un grand nombre de personnes venues de tous les villages. Pour ne donner qu'un exemple, l'un de ces cours contenait deux sujets, " erreurs » et "vieilles croyances", et avait pour but de démontrer que la cosmologie traditionnelle, ainsi que les pratiques chamaniques, sont totalement fausses et trompeuses. Certains de ces livrets sont lus pendant les réunions d'oraison du mardi soir. 
L'un des écrits les plus diffusés (car les autres sont essentiellement réservés aux pasteurs) est celui qui s'intitule " "Oro Tamara". Hymnes et chœurs en pacaás novos et portugais ». Il s'agit d'un recueil de 129 hymnes d'origine nord-américaine, en wari' et en portugais. Trois de ces hymnes sont normalement chantées dans les deux langues pour débuter et pour clore le culte. Ceux du début ne sont pas les mêmes que ceux de la fin. De nombreuses personnes possèdent ce recueil et presque tous connaissent les chants par cœur ${ }^{4}$. Certains - surtout de jeunes hommes lettrés disposant d'un salaire et quelques pasteurs - ont un exemplaire de la Bible en portugais qu'ils emportent avec eux aux cultes.

\section{L'ÉCOLE ET LE MATÉRIEL PÉDAgogiQUe}

Sachant que la catéchèse repose sur le contenu des Écritures saintes, les missionnaires ont, très vite, été confrontés au problème de l'alphabétisation des Indiens. Pour le résoudre, ils ont tenté de susciter chez eux «l'intérêt pour le miracle de la parole écrite » (Johnston 1985, p. 219). Chez les Yanomami du Venezuela, un couple de missionnaires raconte comment il a procédé. Ils ont noté sur une feuille de papier le nom des personnes du groupe présentes, puis les ont répétés selon la forme et l'ordre dans lequel ils avaient été dictés « et tout de suite on entendit dire que le "papier parlait" " (ibid., pp. 220-221; pour d'autres commentaires sur ce même récit, voir Gallois et Grupioni 1999, p. 98).

L'alphabétisation des Wari' a commencé juste après le Contact (soit dans la première moitié des années 1960) et a été réalisée par les missionnaires de la NTM. J'ai peu d'informations sur ce sujet, mais certains de ceux qui ont été alphabétisés pendant cette période sont aujourd'hui devenus pasteurs et sont capables de lire, bien que très lentement, le matériel écrit en wari'.

Dans les années 1970, la FUNAI mit en place des postes indigènes qui comprenaient systématiquement une école. Des professeurs laïcs furent alors engagés pour donner des cours, exclusivement en portugais, à des enfants de moins de douze ans déjà alphabétisés. Ils s'appuyaient sur des livres pédagogiques nationaux, utilisés dans de nombreuses écoles brésiliennes, aussi bien en ville qu'à la campagne. De leur côté, les missionnaires ont continué à enseigner dans des classes d'alphabétisation allant jusqu'à la quarta série (équivalent du CM2 en France). Pour cela, ils avaient produit cinq petits livrets, abondamment illustrés, présentant de nombreuses situations et expressions quotidiennes.

Depuis les années 1990, une réforme de l'éducation qui valorisait les spécificités culturelles a permis de former des professeurs indigènes locaux et de produire un matériel pédagogique spécifique pour chaque groupe ethnique. Tous les villages, même les plus petits, comptent, en général, au moins un professeur local. Les cours sont donnés pour partie en wari', pour partie en portugais. 
Les missionnaires de la NTM continuent, pour leur part, à donner des cours d'alphabétisation dans quelques grands villages seulement.

Grâce au « Projeto Açaí », une série de petits fascicules destinés à être utilisés pendant les cours (en même temps que les autres livres d'école) a pu être produite. Ces fascicules ont pour ambition d'enseigner la réalité locale. Par exemple, en géographie, les élèves étudient les rivières de la région et leur propre système de plantation. Tout comme les livres et livrets du passé, ils sont précieusement gardés sous clé dans l'école et ne sont accessibles qu'au moment des cours. Ne pas avoir accès aux livres en dehors de ces moments-là ne semble pas poser de problème aux Wari'.

\section{LA LECTURE ET L'ÉCRITURE}

Comme je l'ai déjà remarqué à propos d'Abrão, les Wari' ne s'intéressent ni aux journaux ou revues, ni aux autres livres (sur ce même sujet chez les Gapun de Papouasie Nouvelle-Guinée, voir Kulick et Stroud 1990, p. 288).

La Bible en portugais et les livres de leçons bibliques sont les seuls écrits que je les ai vus lire en dehors de l'église ou de la salle de cours. Le contexte de lecture est rarement solitaire. La personne qui lit le fait toujours en s'adressant à quelqu'un, même si cette personne n'y accorde pas d'attention particulière. Dans cette période de renouveau religieux à Rio Negro-Ocaia, il est courant de voir un jeune adulte lire la Bible ou l'un des livres de leçons, à voix haute chez lui, pendant que ses enfants jouent autour de lui. Les contextes de lecture par excellence restent cependant les cultes eux-mêmes et les réunions à l'église pendant les jours de prière ou pendant les classes de catéchisme pour les enfants - récemment mises en place dans certains villages. La différence entre l'église et l'école ne se définit pas seulement par le contenu de ce qui est dit, mais surtout parce que l'église est considérée comme un lieu de lecture, ka noro wa («voir»; on dit "voir le papier ») ${ }^{5}$, alors que l'école est un lieu d'écriture, ka xirao wa (le même verbe est employé pour les peintures corporelles au roucou et au genipa). De fait, les Wari' ne produisent aucun écrit religieux. Les sermons sont toujours improvisés oralement. Pour la majorité de ceux qui sont présents au culte, l'église n'est pas seulement un lieu de lecture, mais aussi un lieu où l'on « écoute » (ka taraju wa) la parole de Dieu : plusieurs prières sont adressées à Dieu pour lui demander qu'il leur accorde de bien écouter, d'écouter pleinement.

L'acte de lire tient un rôle central dans le culte. Dans l'église de chaque village, il y a toujours une table et/ou un pupitre, à la manière des églises en ville. Lorsque le pasteur s'installe face aux autres dans l'église, il dépose sur la table une pochette en plastique contenant les divers documents. Même ceux qui ne savent pas lire et qui vont au pupitre pour parler regardent l'endroit où l'on dépose le papier, comme s'ils étaient en train de lire. Du coup, il n'est pas toujours facile, pendant 
le culte, de distinguer ce qui est lu de ce qui est dit. Comme c'est souvent le cas parmi les peuples sans écriture, la répétition est un trait fondamental du discours oral (voir Schieffelin 2007). Lors de lectures, les Wari' répètent, plusieurs fois, ce qui est écrit, en modifiant parfois certains termes qui sont suivis d'explications improvisées et plus détaillées. Ce qui nous permet de différencier la lecture de l'improvisation n'est pas le papier sur lequel se pose le regard car, dans les deux cas, on le regarde, mais la vitesse et la fluidité de la parole. La lecture est très lente, presque syllabique, alors que la parole qui lui succède, avec des termes identiques, est rapide et fluide. Très souvent, le lecteur maintient le doigt sur le papier, comme pour ne pas perdre le fil de sa lecture. Étant donné la lenteur de la lecture, les répétitions et les explications, un pasteur lit rarement plus d'une page d'un livre de leçons pendant les quarante minutes du culte. Une de ces pages contient aussi bien des citations bibliques que les doctrines des missionnaires. Ces deux types de textes, bien que différenciés sur la page par une typographie différente (des italiques pour les citations bibliques), ne sont jamais distincts pendant la lecture à haute voix, adressée aux fidèles, de sorte que ce que disent les missionnaires est immédiatement compris comme « parole de Dieu ». Les conceptions propres aux pasteurs peuvent aussi être présentées au moment de ces lectures, mais sont davantage repérables grâce à la fluidité de parole.

D'après ce que les missionnaires ont transmis aux Wari', la Bible est un livre dicté par Dieu, et c'est en cela que réside la vérité sur les origines du monde, de ses habitants et sur la bonne conduite morale. Comme les missionnaires, les Wari' chrétiens ont coutume d'affirmer que les principes cosmologiques et les événements décrits dans les mythes de leur groupe sont faux parce qu'ils ne savaient pas écrire et que la transmission orale, surtout d'une génération à l'autre, implique des erreurs et des distorsions. Par exemple, le récit wari' sur le déluge serait faux parce qu'il ne contient pas l'épisode de Noé. En même temps, il confirme, pour les missionnaires, le caractère historique de l'épisode du déluge, ce qui valide la conception fondamentaliste missionnaire de la Bible. En effet, face à ce constat, les Wari' se sont exprimés de la façon suivante : "Ah, c'était donc ça que nos anciens appelaient l'esprit de la pluie ! S'ils avaient su écrire... » ou encore « Les anciens ne disaient pas vrai. C'est ce que disent les Blancs qui est correct». En d'autres occasions, quand je leur demandais pourquoi ils croyaient ${ }^{6}$ en ce que les Blancs disaient et non pas en ce que disaient leurs parents et grands-parents, ils répondaient :

Les anciens ne savaient pas écrire. Les Blancs (wijam, ennemi), eux, écrivent. Les Blancs très, très anciens écrivaient. Une femme est tombée enceinte de Jésus. Nous, on parlait sans raison : tan tan na e', oro maho, taxi tari [mythes]. Nous avions peur des fantômes, peur de couler. C'est seulement quand nous avons entendu ici [ce que les Blancs disaient] que nous avons compris que ce n'était pas ainsi. C'est ça que nous avons pensé [...]. Les anciens disaient que nous allions dans le monde subaquatique [après la mort], que nous devenions des pécaris, que notre âme devenait pécari, que nous sortions... Ils 
ne savaient pas écrire. Depuis que nous sommes devenus croyants, nous mangeons des éperviers, des chouettes, des coatis, tout ce qui est de la nourriture bizarre... du tamanoir. C'est moi qui ai créé tous les animaux [a dit Dieu]. (Paletó, homme d'environ 80 ans)

Quand le pasteur Awo Kamip m'a dit que nous venions de l'argile, que c'est comme cela que nous avons commencé, je lui ai demandé pourquoi nous n'en savions rien avant. Il m'a répondu : « Si seulement on avait su écrire ! ».

Tous les textes n'ont pas le même statut de vérité. L'idée que les textes véritables sont ceux qui ont été inspirés par Dieu - par opposition aux textes pédagogiques de l'école - est devenue tellement évidente aux yeux des Wari' qu'ils sont convaincus que l'écriture prendra de l'importance dans la vie posthume céleste. Selon eux, ce ne sera qu'au ciel qu'ils accèderont véritablement à la maîtrise de l'écriture car c'est là qu'ils pourront écrire sous inspiration divine ${ }^{7}$. Comme l'explique Wao Tokori (homme d'environ 80 ans), « dans le ciel, tout le monde écrit... les vieux, les enfants, les femmes. Dans les maisons, comme ici, on écrit, on écrit. On ne se dit pas: "Allons récolter des vers palmistes, allons chasser !" Tout le monde est repu ». L'écriture est aussi une activité qui caractérise les autres habitants du ciel : Dieu écrit en permanence sur ce que font les Wari', leurs bonnes comme leurs mauvaises actions ${ }^{8}$ (pour une analyse de la conception wari' du ciel, voir Vilaça 2007).

\section{LA MORALE CHRÉTIENNE}

Robbins (2004) remarque que, pour les Urapmin de Papouasie-Nouvelle Guinée, le christianisme a introduit un ensemble de règles morales distinctes des règles traditionnelles, en particulier parce qu'elles ne consistaient pas en règles claires comme le système des tabous. Le péché passait de l'extérieur à l'intérieur de l'individu, du respect des règles et de rituels spécifiques à l'intention de chaque action ou pensée, de sorte que les actions quotidiennes devenaient l'objet d'un examen constant. De manière générale, le conflit est perçu par les Urapmin comme une confrontation entre le désir - qui mène au péché, mais qui est essentiel au mouvement de la vie sociale - et la loi, la soumission et le contrôle - qui assurent la salvation. La cause des infortunes devient un problème interne plutôt qu'une question de respect ou de transgression d'un corpus légal défini. La lecture de la Bible et des textes religieux au sens large a pour fonction de clarifier ces nouvelles règles morales. De cette manière, il est fréquent que les sermons tournent autour d'une « liste de vertus » : cette loi inconnue doit être formulée en permanence pour être compréhensible (ibid., p. 228).

Pour ce qui est des Wari', leur intérêt pour la Bible semble reposer sur deux points centraux : les leçons de morale et le processus de création du monde par Dieu qui les met en position de prédateurs des animaux et, par voie de 
conséquence, en position d'humains (Vilaça 2008, 2009), un thème sur lequel je reviendrai à la fin. Intéressons-nous pour l'instant aux questions morales.

À la différence de ce qui se produit chez les Urapmin, la morale chrétienne n'est pas considérée comme une morale nouvelle, mais comme la confirmation par écrit de celle qui existait déjà chez les Wari'. Le respect de ces règles leur permet d'actualiser ce qu'ils considèrent comme un mode de vie idéal : la vie entre consanguins, réglée par la générosité et surtout par l'absence de colère. C'est ce contenu moral de la Bible - qui produit des relations - qui la différencie des autres livres que les Wari' connaissent et pour lesquels ils ne montrent pas le même intérêt ${ }^{9}$. Comme j'ai tenté de le montrer à d'autres occasions, les Wari' cherchent, dans la vie de tous les jours, à masquer les relations d'affinité qui sont à l'origine de tensions internes (Vilaça 1992, 2006, 2010). Ils le font en ayant recours à des vocatifs de consanguinité pour les affins co-résidents (ou des teknonymes insistant sur la consanguinité en passant par la première génération descendante, comme « père de mon petit-fils », par exemple), et en promouvant leur consanguinité à travers la commensalité. La relation entre parents consanguins se définit par le respect d'une morale spécifique, ancrée dans la valeur positive accordée à la générosité (tout particulièrement alimentaire) et sur la valeur négative liée à la colère. « Être en colère » ( ka mana wa) et « agir de manière colérique » (ka xirakwa) sont les expressions que les Wari' utilisent pour traduire le terme " péché ». La colère est justement ce qui caractérise la relation entre ennemis et les affins sont vus comme des ennemis potentiels. La colère des affins se manifeste de façon concrète par des combats de massue et des actes de sorcellerie, une des causes de mort chez les Wari' ${ }^{10}$.

Ainsi, les Wari' ont été séduits par la morale chrétienne parce qu'elle est fondée sur l'idée de l'amour universel, lequel régit les relations entre membres de la communauté qui se désignent mutuellement comme frères et sœurs. La colère et ses maux sont le thème central des sermons à l'église (et l'un des sujets importants du Nouveau Testament). La morale chrétienne, en confirmant les valeurs morales wari' et en rendant celles-ci constamment explicites à travers la lecture des textes bibliques, représente un moyen supplémentaire - et efficace, puisque fondé sur un écrit, respecté par les Blancs ${ }^{11}$ - pour envisager une vie meilleure dans un monde de consanguins ${ }^{12}$.

Au moment où je les rencontrai en 1986 alors qu'ils n'étaient pas croyants, les Wari' se référaient, avec nostalgie, à leur première conversion dans les années 1970, ils l'évoquaient comme une période de fraternité généralisée marquée par l'absence de colère :

Les étrangers, Wari' des autres sous-groupes, étaient appelés " frère cadet, frère aîné ".

Les frères cadets étaient ceux qui étaient devenus croyants après. (OroNao', homme d'environ 70 ans, résidant à Rio Negro-Ocaia)

Ils avaient arrêté les combats de casse-tête, arrêté d'être en colère. Il n'y avait plus de Wari' en colère, ils étaient tous croyants. (OroEo, homme d'environ 60 ans, résidant au Rio Negro-Ocaia) 
Aujourd'hui, l'expérience de la conversion est exprimée en des termes similaires :

C'est comme ça pour nous. L'esprit de Dieu est arrivé, et il pense à nous. C'est pour ça que nous ne sommes plus constamment en colère les uns contre les autres. C'est pour ça que nous sommes heureux. Il a dit de faire comme ça, pour que nous soyons satisfaits les uns des autres. Si Dieu nous ôte l'entendement, on ne pense pas comme il faut. (Awo Kamip, pasteur)

Autrefois nous n'étions pas contents les uns des autres. Nous avions de la colère les uns envers les autres souvent ; nous ne nous aimions pas. Mais la parole de Dieu est arrivée, et cela nous a rendu la parole joyeuse. (Xin Xoi, pasteur récemment décédé, culte du 27 janvier 2002).

Plus que de rendre possible l'exercice du bien-être collectif, Dieu surveille son exécution et punit, le jour du Jugement dernier, ceux qui se sont opposés à son enseignement :

Il ne nous voit pas? Il nous voit. Il nous entend. Il nous entend médire. Il entend les choses mauvaises que nous disons. C'est Dieu. Il sait. Il sait. Il nous voit aussi, quand nous n'aimons pas nos co-résidents. Il ne nous verrait pas quand nous faisons ça ? Il nous voit, il nous entend [...] Il est bon que vous soyezjoyeux, mes frères. N'ayez pas de haine les uns envers les autres. Il n'aime pas que vous ressentiez de la colère les uns envers les autres [...] Telle est la parole de Dieu. (Xijan, pasteur auxiliaire, 27 Janvier 2002).

Comme pour les Uramin (Robbins 2004), la Bible les intéresse particulièrement parce qu'elle formule clairement ces règles morales. Un jour, j'ai demandé à Abrão pourquoi les Wari' s'intéressaient uniquement à la Bible (et aux textes religieux produits par les missionnaires), et non pas aux autres textes, il m'a répondu :

C'est pour savoir ce qu'on peut et ne peut pas faire, parce qu'il y a tellement de choses qu'on ne peut pas faire. Ils disent : « on ne peut pas voler », " on ne peut pas être fier d'un vêtement neuf ». Cela met des barrières à la pensée, pour penser seulement aux choses bonnes. ${ }^{13}$

Pour résumer, disons que la Bible offre aux Wari' une confirmation, à travers la parole des Blancs - considérés comme tout puissants -, de la validité d'une morale qui est, avant tout, la leur et qui va non seulement permettre de construire une relation avec Dieu, mais surtout qui rend possible l'établissement de relations entre humains.

\section{Manger la parole de Dieu}

Voyons à présent comment la relation qui existe entre ces valeurs morales et la commensalité entraîne une association entre la parole de Dieu et la nourriture. 
Un jour, Wao Tokori (homme d'environ 80 ans) m'a expliqué que, dans le ciel, on écrit tout le temps et que nul ne se préoccupe de nourriture. Quand je lui ai demandé : «Finalement que mange-t-on? », il m'a répondu : «C'est ça qu'ils mangent. Ils mangent le papier. Ils sont repus de Ses écrits ». Dans une conversation qui portait sur le ciel, Paletó (homme du même âge) affirma quelque chose de semblable :

- Qu'est-ce qu'on fait dans le ciel ?

- On écrit beaucoup.

- Qu'est-ce qu'on écrit?

- Je ne sais pas trop. Je crois que c'est la parole de Dieu (oro kapijakon Deus). C'est de ça qu'ils sont repus.

L'association entre la parole de Dieu et la nourriture est fréquente lors des cultes :

C'est ça qui repaît notre esprit. C'est comme si nous mangions la parole de Dieu. (Xin Xoi, pasteur, Rio Negro-Ocaia, sermon pendant un culte filmé)

C'est comme de la nourriture (karawa). Regardez les animaux que nous mangeons. Si nous mangeons les animaux, tous les animaux que nous tuons, nous sommes très repus, ça nous rend plus fort. Si nous n'avions pas de nourriture à manger, si nous passions des jours entiers sans attraper de poisson, il n'y aurait rien à manger. Et on est faible, et triste. On devient tout mou. C'est la même chose aujourd'hui avec la parole de Dieu. C'est pour ça que nous chutons sans arrêt (nous dévions de la morale chrétienne). Nous ne mangeons pas la parole de Dieu. Nous avons avec nous la parole de Dieu sans en profiter, sans la manger. (Awo Tot, pasteur auxiliaire, Rio Negro-Ocaia, 27 Janvier 2002, culte filmé)

Il ne s'agit pas d'une association originale, dans la mesure où la Bible et la doctrine missionnaire sont pleines d'allégories de ce type. Citons par exemple :

Matthieu $4: 4$. Et lui [Jésus] de répondre : Il est écrit : L'homme ne vit pas que de pain mais de toute parole qui sort de la bouche de Dieu [sic].

Jean $6: 35$. Jésus leur dit : je suis le pain de la vie. Qui vient à moi n'aura jamais faim, qui se fie à moi n'aura jamais soif.

Robbins (2004, p. 264) a observé chez les Urapmin que, pour eux - qui, à la différence des Wari', sont pentecôtistes, - « c'est seulement avec l'aide de l'Esprit saint que le pasteur peut faire le sermon qui "alimente" les personnes, les nourrit avec la nourriture spirituelle qui va leur donner la force d'éviter le péché ». Selon ce même auteur, la prière finale des cultes « attire l'attention sur le fait que les personnes ont désormais "mangé" les paroles de Dieu et que, puisqu'elles sont nourries, elles peuvent "sortir" et devront user de cette force acquise à l'instant pour éviter le péché » (ibid., p. 266) ${ }^{14}$.

Bien que les Wari' n'aient rien inventé en associant la parole de Dieu et la nourriture, on peut tout de même affirmer que les missionnaires ont semé leur 
conception en terrain fertile chez les Wari' puisque, pour ceux-ci, la nourriture et l'acte de donner de la nourriture sont des médiateurs fondamentaux dans les relations sociales : ils les constituent et les définissent. Regardons de plus près ce qu'il en est. Les deux termes principaux de l'ontologie wari' sont wari' et karawa. Wari" est le pronom « nous », première personne du pluriel, et signifie « gens », personne », " être humain ». Karawa désigne la nourriture, la proie. Il s'agit de positions qui peuvent être occupées par différents êtres, Wari' ou animaux qui se perçoivent eux-mêmes comme des personnes (pour la notion de "perspectivisme », voir Viveiros de Castro 1996, 2002). Celui qui occupe la position de wari" est celui qui agit comme prédateur, au contraire de karawa qui est donc la proie. L'acte de prédation - et, donc, de se nourrir - est central pour différencier des êtres qui sont, en principe, identiques ou, pour le dire autrement, humains. Celui qui mange est défini, au moment où il le fait, comme humain, celui qui est mangé est considéré comme non-humain, autre, animal (Vilaça 1992, 2000, 2005, 2007). À l'inverse, la commensalité - ou le fait de manger ensemble - est essentielle à la définition de l'identité entre les personnes (ou entre les animaux) : elle favorise les relations de consubstantialité et de consanguinité. Les Wari' disent que ceux qui mangent les mêmes aliments - soit parce qu'ils sont en train de les partager, soit parce qu'ils ont le même régime alimentaire - peuvent devenir parents et égaux. La commensalité, additionnée aux soins quotidiens et à la proximité physique, joue un rôle important dans la constitution de parents. Dans le même ordre d'idée, les chamanes, par exemple, racontent qu'ils mangent avec les animaux avec lesquels leur esprit vit et, quand ils veulent accompagner un autre type d'animal, ils doivent nécessairement changer leur régime alimentaire pour le rendre compatible avec celui de cette nouvelle espèce. Quand je suis arrivée chez les Wari', ils insistaient pour que je mange des vers palmistes, ce qui, dans la vision des Blancs, est propre au régime alimentaire wari'. La fois où j'en ai enfin mangé, ceux qui étaient avec moi dans la forêt sont rentrés au village en disant à tout le monde que j'étais devenue complètement Wari'. De manière analogue, les Wari' disent qu'ils deviennent Blancs parce qu'ils mangent désormais du riz, de l'huile, des haricots et des pâtes (Vilaça 2006, 2010). Selon les Wari', l'acte de manger permet de classer les êtres selon deux catégories qui fondent l'univers wari' : celui à qui l'on offre la nourriture - ou avec qui on la partage - est un égal, un parent, wari' ; celui qui devient nourriture est un autre, animal ou ennemi, karawa. On comprend, dès lors, pourquoi les Wari' insistent tant sur la commensalité pour évoquer leur expérience du christianisme : elle est, pour eux, une expression directe de l'actualisation de bons principes moraux, comme l'illustrent ces propos tenus à l'époque où ils n'étaient plus croyants (années 1980) :

Quand on croyait en Dieu, un homme qui tuait une proie la grillait, la découpait en morceaux et la transportait dans un panier pour aller la distribuer à tout le monde [...]. Tous mangeaient ensemble. Aujourd'hui, on tue la proie et on la mange égoïstement. (OroAt, homme de 70 ans, habitant de Rio Negro-Ocaia) 
Si les OroNao' arrivaient ici, nous les inviterions pour le repas et mangerions tous ensemble dans la joie. Nous chanterions les musiques de Dieu. Nous chanterions, chanterions, jusqu'à ce que nous allions tous manger ensemble. Si Xijan avait de la nourriture, il appellerait tout le monde pour manger dans sa maison. Tout le monde mangerait. Tout le monde mangerait heureux. (OroEo, homme d'environ 50 ans, habitant de Rio Negro-Ocaia)

Il apparaît qu'en condamnant la colère, la parole de Dieu réitère ce que produit la commensalité, à savoir la consanguinité. Comme nous l'avons déjà dit, cela se réplique à différents niveaux : les Wari' sont des commensaux parce qu'ils mangent le même aliment qu'est la parole de Dieu et parce qu'ils partagent les repas entre membres du groupe chrétien ${ }^{15}$. L'effet ne se limite pas seulement à des relations internes. La christianisation joue également un rôle important dans le monde wari' où l'humanité est considérée en position instable, dépendant d'une différenciation entre prédateur et proie, mangeur et mangé, différenciation qui n'est pas donnée a priori, mais est produite par les actes. Certains versets de la Bible qui ont le plus retenu l'attention des Wari' ont pour thème le fait que l'être humain est en position fixe de prédateur.

Après avoir créé le ciel, la terre et les animaux en tous genres, Dieu, selon la version wari' [Genèse 1. 26] : «Dit aussi. Faisons les gens. Qu'ils soient semblables à nous. Ils seront les leaders (taramaxikon) de tous les poissons, oiseaux et de tous les animaux étranges. Ils seront les leaders de toute la terre, également. C'est ce qu'Il dit». [La Bible en français ${ }^{16}$ : « Dieu dit enfin : Faisons les êtres humains ; qu'ils soient comme une image de nous, une image vraiment ressemblante ! Qu'ils soient les maîtres des poissons dans la mer, des oiseaux dans le ciel et sur la terre, des gros animaux et des petites bêtes qui se meuvent au ras du sol! »].

[Genèse 1. 28] : « Content, il dit. Reproduisez-vous beaucoup [...]. Éparpillez-vous sur toutes les autres terres. Soyez les leaders des poissons, des oiseaux et de tous les animaux ». [La Bible en français : « Puis Il les bénit en leur disant : Ayez des enfants, devenez nombreux, peuplez toute la terre et dominez-la ; soyez les maîtres des poissons dans la mer, des oiseaux dans le ciel et de tous les animaux qui se meuvent sur la terre »]

La création selon la Bible, en déterminant que les animaux sont uniquement des proies, les désubjectivise et établit que les Wari' sont prédateurs et, donc, les seuls êtres humains. En acceptant cette perspective chrétienne et en s'identifiant à elle, les positions wari' et karawa que les Wari' devaient assumer dans une relation d'opposition mutuelle deviennent alors des catégories fixes.

Jadis, ils évitaient le tatou, le coati. Lorsque nous avons connu les Blancs, les croyants nous ont dit que nous devions manger de tout, car c'était Dieu qui nous avait faits. Que cela ne rendait pas malade. Des femmes enceintes mangent du tatou, du faucon. Cet animal est devenu un oiseau véritable (une proie) pour nous. Elles mangent des anguilles et il n'arrive rien. Pourquoi ? « J'ai créé les animaux », dit Dieu. " Alors, c'est comme ça », nous avons dit. (Paletó, homme d'environ 80 ans) 


\section{Conclusion}

Nous avons voulu apporter, dans ces lignes, des éléments de compréhension sur l'intérêt que les Wari' manifestent pour le message chrétien de la Bible. Très vite, il est apparu que les Wari', tout comme les missionnaires protestants fondamentalistes de la NTM, considèrent comme un fait véridique et indiscutable que ce texte fut dicté aux hommes directement par Dieu et qu'il s'est conservé intact à travers les temps parce qu'il a été fixé par écrit.

Nous avons également pu constater que la valeur de vérité accordée à l'écriture ne s'applique pas aux autres types de documents, comme les journaux, les revues, les livres didactiques utilisés à l'école et abordant des thèmes relevant de la réalité locale (cours d'eau, systèmes de plantation, rituels, mythes, entre autres). Pour les Wari', en effet, ces documents n'ont pas d'intérêt particulier, ne sont pas des sujets de discussions entre eux et restent dans les locaux de l'école.

Par ailleurs, les textes, comme la Bible ou les leçons produites par les missionnaires, traduits soit en wari', soit en portugais, sont lus aussi bien pendant un culte que pendant les activités quotidiennes de la famille. Le contenu de la Bible offre aux Wari' une solution, même si elle n'est que provisoire (ce qui pourrait, d'ailleurs, expliquer leur inconstance dans la conversion), aux difficultés qui transparaissent dans le domaine de l'expérience et, notamment, à l'instabilité du statut d'être humain qui les rend constamment sujets aux transformations sous l'effet de la maladie et de la mort. Deux thèmes (et les épisodes qui leur sont liés) sont retenus par les Wari' : la moralité liée aux histoires familiales ou «tribales » et la création du monde racontée dans la Genèse (premier livre qui ait été traduit en langue wari' par les missionnaires, selon les Wari'). Le premier thème met l'accent sur les relations de fraternité et de générosité qui correspondent à un idéal chez les Wari', idéal dans lequel ils seraient un groupe humain stable, non soumis au potentiel d'altération due à l'affinité qui s'exprime surtout par le biais de la sorcellerie. Pour sa part, l'épisode de la création établit clairement une différenciation entre les Wari' et les animaux, ce qui permet aux premiers d'être définitivement fixés dans une position de prédateurs qui équivaut, dans leur pensée, à celle d'être humain. Nous avons aussi montré l'importance pour les Wari' de la commensalité pour tout ce qui touche aux relations de parenté, tout particulièrement à celle de fraternité. Il n'est donc pas étonnant de les voir s'approprier l'association - très courante dans d'autres contextes culturels - entre parole divine et nourriture. Nourris par Dieu, les gens sont considérés comme ses enfants et deviennent donc, entre eux, frères et sœurs. Cette fraternité est renforcée par la commensalité qui se met en place au moment de la lecture de la Bible et qui prend la forme d'une distribution généreuse d'aliments, ainsi que nous l'a fait observer un homme que nous avons pu interroger.

Par conséquent, l'intérêt des Wari' pour la Bible et pour la parole écrite réside essentiellement dans son potentiel relationnel, c'est-à-dire dans l'effet qu'elle peut 
avoir sur les relations entre les personnes, soit directement, soit par exclusion des animaux de cet univers relationnel. Dans un certain sens, et d'après ce que nous avons pu observer pour la commensalité, nous pouvons dire que le statut de vérité de l'écriture dépend de sa valeur alimentaire. *

* Manuscrit reçu en décembre 2009, accepté pour publication en décembre 2010.

\section{Notes}

Ce texte est une version modifiée d'une présentation faite dans le cadre du séminaire lié au projet Capes-Cofecub «Arte, imagem e memória: horizontes de uma antropologia da imagem e da cognição ». Je remercie Carlo Severi et tous les participants du séminaire pour leurs commentaires, ainsi que les lecteurs anonymes du Journal de la Société des Américanistes.

1. Les Wari' se divisent en six groupes dénommés OroNao', OroEo, OroAt, OroMon, OroWaram et OroWaramXijein.

2. À propos de «l'inconstance de l'âme sauvage », voir Viveiros de Castro $(1992,2002)$ pour les Tupinambá du XVI ${ }^{\mathrm{e}}$ siècle et Vilaça $(1996,2002)$ pour les Wari'.

3. Pour un exemple de population autochtone qui s'intéresse aux événements de la fin du monde, voir le travail de Robbins (2004) chez les Urapmin.

4. Il s'agit d'un répertoire fixe, fourni par les missionnaires. Ce n'est que récemment que certaines personnes - souvent les plus scolarisées, parmi lesquelles on peut compter Abrão - ont commencé à composer des hymnes qui consistent en des mises en musique de versets des évangiles, suivant la mélodie typique des hymnes. D'après ce que j'ai pu constater, ces hymnes composées ne sont pas transcrites sur papier, mais directement enregistrés sur cassette.

5. Bien que la notion wari' de « voir » soit en étroite relation avec le chamanisme (le chamane étant "celui qui voit»), on ne peut pas soutenir que les Wari' conçoivent la lecture comme un acte chamanique, à la manière des Piro étudiés par Gow (2001). Ils ne conçoivent pas non plus que la lecture de la Bible leur permette de faire une expérience directe de Dieu, comme dans une « conversation » à la façon des évangélistes.

6. Howa : croire en ce que dit quelqu'un, prêter foi, et non pas croire en l'existence de quelque chose. Voir Pouillon (1979), Robbins (2007), Basso (1987, p. 238, qui observe que, pour les Kalapalo, la vérité dépend moins de la relation entre proposition et fait que de la relation entre interlocuteurs) et Figueiredo (2010, pp. 302, 309).

7. Cela ne signifie pas pour autant que les Wari' ne savent pas écrire. Comme cela a été observé chez les Gapun (Kulick et Stroud 1990), l'écriture est utilisée uniquement pour rédiger des billets qui circulent localement et qui constituent un mode indirect de déclaration (entre couples d'amoureux) et de sollicitation de cadeaux.

8. Il convient de rappeler que l'association entre vie posthume et activité d'écriture n'est pas propre aux Wari' : chez les Gapun de Papouasie-Nouvelle Guinée, selon Kulick et Stroud (1990, p. 301, note 3), après la mort, les humains, avant d'aller au ciel, passent quelque temps à Rome pour y apprendre à écrire.

9. Kulick (1992, p. 177) raconte que, chez les Gapun de Papouasie-Nouvelle Guinée, quand ces derniers commentent l'expérience scolaire, ils évoquent les « événements drôles et scandaleux qui ont lieu là [à l'école] » et non pas l'apprentissage de la « culture » européenne - si ce n'est dans le sens de secrets qui, dévoilés, permettraient d'accéder aux richesses des Européens.

10. L'adoption du christianisme dans le but d'éradiquer la sorcellerie se retrouve dans de nombreux groupes autochtones. Pour l'Amazonie, voir Wright (1998); pour l'Afrique, où la sorcellerie est un thème central, voir Douglas (1999) et Meyer (1998, 1999).

11. Notons que le christianisme permet aussi une relation de germanité (donc d'égalité) avec les chrétiens blancs (voir Robbins 2004 pour les Urapmin). 
12. La même idée de conversion comme expérience collective du bien-vivre se retrouve chez les Piro (Gow 2006, p. 218).

13. Si l'on considère que les leçons de morale biblique sont très souvent implicites dans les événements de la vie familiale ou d'une unité sociale délimitée et si l'on considère également que ces groupes sont très préoccupés par les relations interpersonnelles (Wagner 1975, pp. 24-26), il devient alors nécessaire de tenir compte des spécificités de la narration biblique pour tenter d'évaluer l'intérêt qu'ils portent à ce livre.

14. Voir Coleman (2006, p. 170) à propos des Faith Christians de Suède et Rutherford (2006, p. 251, note 14) qui a observé que, chez les Biaks d'Indonésie, certaines musiques « comparent la parole de Dieu à un délicieux poisson boucané ».

15. Notons que, bien que l'aliment offert fasse partie de la personne qui l'offre, l'acte d'ingérer les sépare définitivement. En ce sens, ingérer la parole de Dieu n'est pas un acte de cannibalisme, mais c'est, avant tout, un geste de commensalité entre les humains et Dieu. Je remercie l'un des lecteurs du Journal de la Société des Américanistes de m'avoir permis d'éclaircir ce point.

16. Voir la traduction en français sur le site http://www.interbible.org/interBible/ecritures/bu/ index.php, consulté le 11/05/2012 (Bible en français courant, Société biblique française, 1997).

\title{
RÉFÉRENCES CITÉES
}

\author{
BASSO Ellen \\ 1987 In favor of deceit. A study of tricksters in an Amazonian society, University \\ of Arizona Press, Tucson.
}

Coleman Simon

2006 "Materializing the self. Words and gifts in the construction of charismatic Protestant identity », in Fenella Cannell (éd.), The anthropology of Christianity, Duke University Press, Durham/Londres, pp. 163-184.

Crapanzano Vincent

2000 Serving the Word. Literalism in America from the pulpit to the bench, The New Press, New York.

\section{Dawson Millie}

2000 All day long. Missionaries reaching tribes in the Amazon, Winepress Publishing, Enumclaw.

Douglas Mary

1999 "Os Lele revisitados, 1987 acusações de feitiçaria à solta », Mana. Estudos de Antropologia Social, 5 (2), pp. 7-30.

FERNANDES Rubem César

1980 "Um exército de anjos : as razões da Missão Novas Tribos », Religião e Sociedade, 6, pp. 129-166.

FigueIREDo Marina

2010 A flecha do ciúme. O parentesco e seu avesso entre os Aweti do Alto Xingu, thèse de doctorat, PPGAS/MN/UFRJ, Rio de Janeiro. 
Gallois Dominique et Luis Donisete GruPIONI

1999 «O Indio na Missão Novas Tribos », in Robin Wright (éd.) Transformando os Deuses. Os múltiplos sentidos da conversão entre os povos indígenas no Brasil, Editora da Unicamp, Campinas, pp. 77-129.

Gow Peter

$2001 \quad$ An Amazonian myth and its history, Oxford University Press, Oxford.

2006 «Forgetting conversion. The Summer Institute of Linguistic mission in the Piro lived world », in Fenella Cannel (éd.), The anthropology of Christianity, Duke University Press, Durham/Londres, pp. 211-239.

HARDING Susan

2001 "Representing fundamentalism : the problem of the repugnant other », Social Research, 58 (2), pp. 373-393.

JOHNSTON Kenneth

1985 The story of the New Tribes Mission, New Tribes Mission, Sanford.

KuLICK Don

1992 Language shift and cultural reproduction. Socialization, self, and syncretism in a Papua New Guinean village, Cambridge University Press, Cambridge.

Kulick Don et Christopher STROud

1990 "Christianity, cargo and ideas of self : patterns of literacy in a Papua New Guinean village », Man (n. s.), 25 (2), pp. 286-304.

McIlwain Trevor

2003 Alicerces firmes : da criação até Cristo, Missão Novas Tribos do Brasil, Anápolis [1991 pour l'édition anglaise].

MeYer Birgit

1998 "Make a complete break with the past" : memory and postcolonial modernity in Ghanaian pentecostal discourse ", in Richard Werbner (éd.), Memory and the postcolony : African anthropology and the critique of power, Zed Books, Londres, pp. 182-208.

1999 Translating the devil. Religion and modernity among the Ewe in Ghana, Edinburgh University Press for the International African Institute, Édimbourg.

Pouillon Jean

1979 « Remarques sur le verbe "croire” », in Michel Izard et Pierre Smith (éd.), La fonction symbolique, Gallimard, Paris.

RoBbins Joel

2004 Becoming sinners. Christianity and moral torment in a Papua New Guinea society, University of California Press, Berkeley/Los Angeles/Londres.

2007 "Continuity thinking and Christian culture », Current Anthropology, 48 (1), pp. 5-38.

RUTHERFORD Danilyn

2006 "The Bible meets the idol. Writing and conversion in Biak, Irian Jaya, Indonesia ", in Fenella Cannell (éd.), The anthropology of Christianity, Duke University Press, Durham/Londres, pp. 240-272. 
SCHIEFFELIN Bambi

2007 «Found in translating : reflexive language across time and texts in Bosavi, Papua New Guinea », in Miki Makihara et Bambi Schieffelin (éd.), Consequences of contact. Language ideologies and sociocultural transformations in Pacific societies, Oxford University Press, Oxford.

SEEGER Anthony

1980 Os índios e nós, Campus, Rio de Janeiro, pp. 43-57.

1981 Nature and society in central Brazil. The Suya Indians of Mato Grosso, Harvard University Press, Cambridge Mass./Londres.

Segal Alan

2003 «Text translation as a prelude for soul translation », in Paula Rubel et Abraham Rosman (éd.), Translating cultures. Perspectives on translation and anthropology, Berg, Oxford/New York.

VILAÇA Aparecida

1992 Comendo como gente : formas do canibalismo wari', Editora UFRJ/Anpocs, Rio de Janeiro.

1996 «Cristãos sem fé : alguns aspectos da conversão dos Wari' (Pakaa-Nova) », Mana. Estudos de Antropologia Social, 2 (1), pp. 109-137.

1997 "Christians without faith : some aspects of the conversion of the Wari' ", Ethnos, 62 (1-2), pp. 91-115.

2000 «Relations between funerary cannibalism and warfare cannibalism : the question of predation », Ethnos, 65 (1), pp. 83-106.

2002 "Missions et conversions chez les Wari' : entre protestantisme et catholicisme ", L'Homme, 164, pp. 57-79.

2005 "Chronically unstable bodies. Reflexions on Amazonian corporalities », The Journal of the Royal Anthropological Institute, 11 (3), pp. 445-464.

2006 Quem somos nós. Os Wari'encontram os brancos, Editora UFRJ, Rio de Janeiro.

2007 "Cultural change as body metamorphosis », in Carlos Fausto et Michael Heckenberger (éd.), Time and memory in Indigenous Amazonia anthropological perspectives, University Press of Florida, Gainesville, pp. 169-193.

2008 «Conversão, predação e perspectiva », Mana. Estudos de Antropologia Social, 14 (1), pp. 173-204.

2009 "Conversion, predation and perspective », in Aparecida Vilaça et Robin Wright (éd.), Native Christians. Modes and effects of Christianity among Indigenous peoples of the Americas, Ashgate, Burlington, pp. 147-166.

2010 Strange enemies : Indigenous agency and scenes of encounters in Amazonia, Duke University Press, Durham/Londres.

Viveiros de CASTRo Eduardo

1992 «O mármore e a murta : sobre a inconstância da alma selvagem », Revista de Antropologia, 35, pp. 21-74.

1996 «Os pronomes cosmológicos e o perspectivismo ameríndio », Mana. Estudos de Antropologia Social, 2 (2), pp. 115-143.

2002 A inconstância da alma selvagem, Cosac \& Naify, São Paulo. 
WAGNER Roy

1975 The invention of culture, Prentice-Hall, New Jersey.

WRIGHT Robin

1998 Cosmos, self, and history in Baniwa religion : for those unborn, University of Texas Press, Austin. 